\title{
PRODUCTIVE SETS
}

\author{
BY \\ J. C. E. DEKKER
}

Introduction. We shall be concerned with non-negative integers (called numbers), collections of numbers (called sets), and collections of sets (called classes). $\alpha$ is a subset of $\beta$ is denoted by " $\alpha \subset \beta$, , $\alpha$ is a proper subset of $\beta$ by " $\alpha C_{+} \beta$." A function $f(n)$ which maps every number on a number is called effective if there exists a general method which enables us to compute the value of the function for any given value of the argument in a finite number of steps. Similarly an effective function of two variables is defined. A set is called effectively enumerable (e.e.), if it is either empty or the range of an effective function of one variable. A set $\alpha$ is called productive if there exists a general method which enables us to find for any given e.e. subset $\beta$ of $\alpha$ an element $b \in \alpha-\beta$. It follows that no productive set is e.e. The importance of e.e. sets for the foundations of mathematics was shown by Post [4, pp. 287, 288]. One of the concepts he introduced was that of a creative set; this is a set which is e.e. and has a productive complement. If the complement of every productive set were e.e., creative and productive sets could only be studied together. We shall find, however, that there are nondenumerably many productive sets without an e.e. complement. This opens the road to a separate discussion of productive sets. We shall now try to justify such a discussion by pointing out another context in which these sets arise.

Let $C$ be the collection of all real numbers in the interval $(0,1]$ whose nonterminating decimal expansion $0 . x_{0} x_{1}, \cdots$ has the property that $x_{n}$ is an effective function of $n$. Since it can be proved that there are only denumerably many effective functions, it follows that $C$ is denumerable. The nonempty subcollection $A$ of $C$ is called effectively enumerable if there exists an effective function $a_{m n}$ of $m$ and $n$ such that

$$
A=\left\{0 . a_{00} a_{01} \cdots, 0 . a_{10} a_{11} \cdots, \cdots\right\} .
$$

The same diagonal procedure used by Cantor to prove that the set of all real numbers in $(0,1]$ is not denumerable can now be used to prove that $C$ is not effectively enumerable. In fact, if the subclass $A=\left\{0 . a_{00} a_{01} \cdots\right.$, $\left.0 . a_{10} a_{11} \cdots, \cdots\right\}$ of $C$ is given (i.e., given by the effective function $a_{m n}$ ), we can effectively find a real number $\beta \in C-A$ by putting

$$
b_{n}=d_{d f}\left\{\begin{array}{lll}
1 & \text { if } & a_{n n} \neq 1, \\
2 & \text { if } & a_{n n}=1,
\end{array} \quad \beta={ }_{d f} 0 . b_{0} b_{1} \cdots .\right.
$$

Presented to the Society, November 29, 1952; received by the editors September 10, 1953. 
The analogy between this property of $C$ and the defining property of a productive set is obvious. There exists therefore a close connection between productive sets and the effective analogue of Cantor's diagonal procedure.

Let $B$ be the class of all e.e. subsets of a set $\alpha$, then $\alpha$ is not e.e. if and only if $\alpha \neq \beta$ for every $\beta \in B$. The fact that $\alpha$ is productive means that given any $\beta \in B$ we can effectively find an element $b \in \alpha-\beta$. Thus a productive set can be considered as a set which not only is not e.e., but of which we can effectively prove that it is not e.e. For, given any e.e. subset $\beta$ of $\alpha$, we can prove $\beta \neq \alpha$ by actually exhibiting an element belonging to $\alpha-\beta$.

Though we have never defined what we mean by "an effective proof that a set is not e.e.," it is clear that the intuitive content of this phrase is sufficiently unequivocal to warrant the statement made in the preceding paragraph that if $\alpha$ is productive there exists an effective proof of the fact that $\alpha$ is not e.e. But it would not be justified to consider the productive sets as the only sets with this property. For suppose we call $\gamma$ a superset of $\alpha$ if $\alpha$ is a subset of $\gamma$ and suppose further that $G$ is the class of all e.e. supersets of $\alpha$. Then $\alpha$ is not e.e. if and only if $\alpha \neq \gamma$ for every $\gamma \in G$. The set $\alpha$ is called contraproductive if there exists a general method which enables us to find for any given e.e. superset $\gamma$ of $\alpha$ an element $c \in \gamma-\alpha$. If $\alpha$ is contraproductive we can prove $\alpha \notin G$ by exhibiting an element $c \in \gamma-\alpha$ for any given set $\gamma \in G$. Since there is no reason to favor subsets of $\alpha$ over supersets of $\alpha$, it follows that a contraproductive set also qualifies as a set for which it can be effectively proved that it is not e.e. And we shall find in section III that there are contraproductive sets which are not productive.

The set $\alpha$ is called semiproductive if there exists a general method which enables us to find for any given e.e. subset $\beta$ of $\alpha$ an e.e. set $\gamma$ such that $\beta C+\gamma \subset \alpha$. It will be shown in section IV that the class of all productive sets is properly included in the class of all semiproductive sets. Using a precise definition of the phrase "an effective proof that a set is not e.e." one could investigate whether or not such a proof could exist for a set which is semiproductive, but not productive. We shall, however, not attempt to propose such a definition. The main purpose of this paper is to give precise definitions of a productive set and a few related concepts and to investigate some of their properties. This is made possible by the fact that there exists a technical concept which is considered by most logicians as equivalent to the intuitive concept of an effective function, namely that of a recursive function.

Preliminaries. The reader is assumed to be familiar with the following concepts: partial recursive function, recursive function, primitive recursive function, recursively enumerable (r.e.) set, recursive set. Productive sets are defined informally in the introduction and formally in section II; a r.e. set with a productive complement is called creative. A set is called immune if it is infinite but has no infinite r.e. subset; a r.e. set with an immune complement is called simple. 
Numbers and functions are denoted by small Latin letters, sets by small Greek letters, and classes by capital Latin letters. The Boolean operations are denoted by " + " for addition, "." or juxtaposition for multiplication, and "' " for complementation. The set $\alpha \beta^{\prime}$ is also denoted by " $\alpha-\beta$ ". In the composition of two or more functions association is to the right. Thus " $f g(n)$ " denotes the same function as " $f(g(n))$." We shall use the well-known primitive recursive function $j(x, y)$ defined by $j(x, y)={ }_{d f} x+(x+y)(x+y+1) / 2$. We shall also employ the following abbreviations:

$\epsilon={ }_{d f}$ the set of all numbers,

$o={ }_{d f}$ the empty set,

$\left\{n_{0}, \cdots, n_{k}\right\}={ }_{d f}$ the set consisting of $n_{0}, \cdots, n_{k}$,

$P={ }_{d f}$ the class of all sets with a finite complement,

$Q={ }_{d f}$ the class of all finite sets,

$E={ }_{d f}$ the class of all recursive sets,

$F={ }_{d f}$ the class of all r.e. sets.

We recall that $P+Q C_{+} E C_{+} F$. Moreover, $E$ is a Boolean algebra and $F$ a distributive lattice with $o$ as null element and $\epsilon$ as one element.

Kleene proved $\left[3\right.$, p. 53] that any partial recursive function $f\left(x_{1}, \cdots, x_{n}\right)$ can be expressed in normal form, i.e., in the form:

$$
f\left(x_{1}, \cdots, x_{n}\right)=l\left[\mu y\left\{h\left(x_{1}, \cdots, x_{n}, y\right)=0\right\}\right],
$$

where $l(x)$ and $h\left(x_{1}, \cdots, x_{n+1}\right)$ are primitive recursive. Let $f(x)$ $=l[\mu y\{h(x, y)=0\}]$, where $l(x)$ and $h(x, y)$ are primitive recursive. Suppose $k$ is any number and $m_{k}=\mu y\{h(k, y)=0\}$; then $m_{k}$ is defined if and only if $f(k)$ is defined. The steps in the computation of $f(k)$ (with respect to this particular expression in normal form) are now defined as follows $\left(^{(1)}\right.$ :

step $n={ }_{d f}\left\{\begin{array}{c}\text { the computation of } h(k, n) \text {, unless } f(k) \text { is defined and } n=m_{k}, \\ \text { the computation of } h(k, n) \text { and of } f(k)=l\left(m_{k}\right) \text {, in case } f(k) \text { is } \\ \text { defined and } n=m_{k} .\end{array}\right.$ Step $n$ in the computation of $f(k)$ is denoted by " $f\langle k ; n\rangle$ "; this step is called successful and is said to produce $f(k)$ if $f(k)$ is defined and $n=m_{k}$.

Following Rice [5, pp. 358, 359] we shall use Kleene's Gödel enumeration of the partial recursive functions of one variable for the characterization of r.e. sets. It easily follows from Kleene's results that there exists a partial recursive function $\Phi(n, x)$ such that:

(1) for any partial recursive function $f(x)$ there exist infinitely many $n$ such that $f(x)=\Phi(n, x)$ and for any partial recursive function $f(x)$ we can find an infinite r.e. set of such numbers $n$,

(2) $\Phi(0, x)$ is defined for no value of $x$ and $\Phi(1, x)$ is the identity function.

(1) This definition is slightly different from that given in $[1$, p. 78]. The advantage of the present definition is that the $n$th step in the computation of $f(k)$ is now defined for all values of $n$ and $k$. As a result " $f\langle k ; n\rangle$ " always denotes a computation though it may be a superfluous one, namely if $f(k)$ is defined and $n>m_{k}$. 
There are obviously many such functions $\Phi(n, x)$, but from now on " $g(n, x)$ " or " $g_{n}(x)$ " will denote one specific function of this type.

We now define: $\omega_{n}={ }_{d f}$ the range of $g_{n}(x)$. It follows that $\left\{\omega_{n}\right\}$ is a sequence of r.e. sets in which every r.e. set occurs infinitely many times. This sequence plays the role in our paper which the sequence $B_{1}, B_{2}, \cdots$ of bases of normal systems plays in Post's paper [4]. If $\alpha$ is a r.e. set every number $n$ such that $\alpha=\omega_{n}$ is called an index of $\alpha$. Thus 0 is an index of $o, 1$ is an index of $\epsilon$; moreover, for every r.e. set $\alpha$ we can find an infinite r.e. set (hence also an infinite recursive set) of indices.

With every partial recursive function $f(x)$ we associate four sets in the following manner:

$$
\begin{aligned}
& \delta(f)={ }_{d f} \text { the domain of } f(x), \\
& \rho(f)={ }_{d f} \text { the range of } f(x), \\
& \gamma(f)={ }_{d f}\left\{f(x) \mid f(x) \in \omega_{x}\right\}, \\
& \chi(f)={ }_{d f}\left\{f(x) \mid f(x) \notin \omega_{x}\right\} .
\end{aligned}
$$

The complements of these four sets are denoted by $\delta^{\prime}(f), \rho^{\prime}(f), \gamma^{\prime}(f), \chi^{\prime}(f)$ respectively. The sets $\delta(f)$ and $\rho(f)$ are obviously r.e. for every partial recursive function $f(x)$; also $\rho(f)=\gamma(f)+\chi(f)$. It can be easily verified (cf. [4, p. 293, lines 20-26]) that there exist recursive functions $i_{n}$ and $k_{n}$ such that all ordered pairs $(i, k)$ with the property $k \in \omega_{i}$ are effectively generated in the sequence

$$
\left(i_{0}, k_{0}\right),\left(i_{1}, k_{1}\right), \cdots \text {. }
$$

Now suppose $f(x)$ is recursive. By comparing the sequence (1) with the sequence

$$
(0, f(0)),(1, f(1)), \cdots
$$

we can effectively generate all ordered pairs which these two sequences have in common. Thus $\gamma(f)$ is r.e. for every recursive function $f(x)$. This argument requires only a minor modification to establish the fact that $\gamma(f)$ is r.e. for every partial recursive function $f(x)$.

\section{COMPletely PRODUCTIVE SETS}

Definition. The set $\alpha$ is completely productive (c.p.) if there exists a recursive function $f(n)$ such that for all $n$,

$$
f(n) \in\left(\alpha-\omega_{n}\right)+\left(\omega_{n}-\alpha\right) .
$$

If the recursive function $f(n)$ is related to $\alpha$ by (1), we say that $\alpha$ is c.p. relative to $f(n)$ and $f(n)$ is called a c.p. function of $\alpha$.

REMARK. Observing that $\alpha \neq \omega_{n}$ if and only if $\left(\alpha-\omega_{n}\right)+\left(\omega_{n}-\alpha\right) \neq 0$, we see that a c.p. set $\alpha$ is characterized by the property that for any given r.e. set $\omega_{n}$ we can prove $\alpha \neq \omega_{n}$ by actually exhibiting a number belonging to the 
symmetric difference of $\alpha$ and $\omega_{n}$. As an example of a c.p. set we mention $\left\{n \mid n \notin \omega_{n}\right\}$ which is c.p. relative to the identity function.

DEFinition. The recursive function $f(n)$ is regular if, for all $k$ and $l$,

$$
f(k)=f(l) \rightarrow f(k) \in \omega_{k} \cdot \omega_{l}+\omega_{k}^{\prime} \cdot \omega_{l}^{\prime} .
$$

Condition (2) can also be expressed as $\gamma(f) \cdot \chi(f)=0$. Every 1-1 recursive function is obviously regular. That the converse is false can be seen as follows. Let $\delta$ be an infinite recursive set of indices of $\epsilon$ and $f(n)$ a 1-1 recursive function. Suppose $h(n)=_{d f} f(n)$ for $n \notin \delta$ and $h(n)=_{d f} 0$ for $n \in \delta$. Then $h(n)$ is a regular function which maps infinitely many numbers on 0 .

We shall now discuss how all c.p. sets can be characterized in terms of regular functions.

THEOREM T 1.1. (1) If $\alpha$ is c.p. relative to $f(n)$, then $f(n)$ is regular and $\chi(f) \subset \alpha \subset \gamma^{\prime}(f)$.

(2) If $f(n)$ is a regular function and $\chi(f) \subset \alpha \subset \gamma^{\prime}(f)$, then $\alpha$ is c.p. relative to $f(n)$.

Proof. (1) Let

$$
f(n) \in \alpha \cdot \omega_{n}^{\prime}+\alpha^{\prime} \cdot \omega_{n} .
$$

Suppose $k \in \chi(f)$, say $k=f(r) \notin \omega_{r}$, then $k \in \omega_{r}^{\prime}$; this implies $k \in \alpha$ by (3). Hence $\chi(f) \subset \alpha$. Now suppose $l \in \gamma(f)$, say $l=f(s) \in \omega_{s}$; this implies $l \in \alpha^{\prime}$ by (3). Hence $\gamma(f) \subset \alpha^{\prime}$, i.e., $\alpha \subset \gamma^{\prime}(f)$. The condition $\chi(f) \subset \alpha \subset \gamma^{\prime}(f)$ implies that $f(n)$ is regular.

(2) Let $f(n)$ be regular and

$$
\chi(f) \subset \alpha \subset \gamma^{\prime}(f) .
$$

For any number $m$, either $f(m) \in \omega_{m}$ or $f(m) \notin \omega_{m}$. By (4) the first possibility implies $f(m) \in \alpha^{\prime}$ and the second one $f(m) \in \alpha$. In both cases $f(m) \in \alpha^{\prime} \cdot \omega_{m}$ $+\alpha \cdot \omega_{m}^{\prime}$.

COROLLARY 1. The sets c.p. relative to the regular function $f(n)$ are exactly the sets of the form $\chi(f)+\tau$, where $\tau \subset \rho^{\prime}(f)$. A mong these sets there is a largest one, namely $\gamma^{\prime}(f)$, and a smallest one, namely $\chi(f)$. If $t$ is the cardinal number of $\rho^{\prime}(f)$, there are exactly $2^{t}$ sets which are c.p. relative to $f(n)$.

Proof. The first statement follows from

$$
\chi(f) \subset \alpha \subset \gamma^{\prime}(f), \quad \rho(f)=\gamma(f)+\chi(f), \quad \gamma(f) \cdot \chi(f)=o .
$$

The second statement follows immediately from the first. The third statement follows from the first, if we take into account that since $\chi(f)$ and $\tau$ are disjoint, different choices of $\tau$ yield different sets $\chi(f)+\tau$.

COROLlary 2. There are exactly c c.p. sets. 
Proof. Let $f(n)={ }_{d f} 2 n$; then $f(n)$ is a regular function and $\rho^{\prime}(f)$ is denumerable. Thus there exist $c$ sets c.p. relative to $f(n)$. It follows that there are at least $c$ c.p. sets. Since there are only $c$ sets in total, we conclude that there are exactly $c$ c.p. sets.

COROLlaRY 3. Every infinite r.e. set can be decomposed into a creative and a c.p. set.

Proof. Let $a(n)$ be a 1-1 recursive function ranging over $\alpha$. Then $\alpha=\gamma(a)$ $+\chi(a)$, where $\chi(a)$ is c.p. relative to $a(n)$. Thus $\gamma^{\prime}(a)$ is c.p., hence also productive. Consequently $\gamma(a)$ is creative, since $\gamma(a)$ is r.e. and $\gamma^{\prime}(a)$ productive.

It follows from Corollary 1 that the number of sets c.p. relative to the regular function $f(n)$ is finite if and only if $\rho(f) \in P$. In particular there exists only one such set in case $\rho(f)=\epsilon$. It is also readily seen from the theorem itself that every c.p. set is included in a c.p. set with a r.e. (hence creative) complement; for if $\alpha$ is c.p. relative to $f(n)$, then $\alpha \subset \gamma^{\prime}(f)$.

In view of the fact that there are only denumerably many r.e. sets, there are also only denumerably many sets with a r.e. complement. Thus, among the $c$ c.p. sets at most denumerably many have a r.e. complement. This establishes the existence of c.p. sets whose complement is not r.e. The proof of the first part of the following theorem can be considered as a more constructive proof of the existence of such sets.

THEOREM T 1.2. (1) There exists a set $\alpha$ such that both $\alpha$ and $\alpha^{\prime}$ are c.p.

(2) $\epsilon$ can be expressed as the sum of $k$ mutually disjoint c.p. sets for every number $k \geqq 2$ and for $k=\boldsymbol{\aleph}_{0}$.

Proof. (1) Let $f_{0}(n)={ }_{d f} 2 n, f_{1}(n)={ }_{d f} 2 n+1$. We denote the sets $\gamma\left(f_{0}\right)$, $\chi\left(f_{0}\right), \gamma\left(f_{1}\right), \chi\left(f_{1}\right)$ by $\gamma_{0}, \chi_{0}, \gamma_{1}, \chi_{1}$ respectively. Then $\gamma_{0}+\chi_{0}+\gamma_{1}+\chi_{1}$ is a decomposition of $\epsilon$ into mutually disjoint sets. Let $\alpha_{0}={ }_{d f} \chi_{0}+\gamma_{1}, \alpha_{1}={ }_{d f} \chi_{1}+\gamma_{0}$, then $\alpha_{0}=\alpha_{1}^{\prime}$ and $\alpha_{0}$ and $\alpha_{1}$ are c.p. relative to $f_{0}(n)$ and $f_{1}(n)$ respectively, by the second part of $\mathrm{T} 1.1$.

(2) Let $k$ be finite and $k \geqq 2, f_{i}(n)={ }_{d f} k n+i, \gamma_{i}=\gamma\left(f_{i}\right), \chi_{i}=\chi\left(f_{i}\right)$, for $i=0, \cdots, k-1$. Then $\gamma_{0}+\chi_{0}+\gamma_{1}+\chi_{1}+\cdots+\gamma_{k-1}+\chi_{k-1}$ is a decomposition of $\epsilon$ into mutually disjoint sets. Let $\alpha_{0}=d_{d f} \chi_{0}+\gamma_{1}, \alpha_{1}={ }_{d f} \chi_{1}+\gamma_{0}$ $+\gamma_{2}+\gamma_{3}+\cdots+\gamma_{k-1}$ and $\alpha_{r}={ }_{d f} \chi_{r}$ for $r=2, \cdots, k-1$. Then the sets $\alpha_{0}, \cdots, \alpha_{k-1}$ satisfy the requirements. We finally consider the case $k=\boldsymbol{\aleph}_{0}$. Let $j_{x}(y)=_{d f} j(x, y), \gamma_{x}={ }_{d f} \gamma\left(j_{x}\right), \chi_{x}=d_{d f} \chi\left(j_{x}\right)$. Then $\gamma_{0}+\chi_{0}+\gamma_{1}+\chi_{1}+\cdots$ is a decomposition of $\epsilon$ into mutually disjoint sets. Let $\alpha_{0}={ }_{d f} \chi_{0}+\gamma_{1}, \alpha_{1}={ }_{d f} \chi_{1}$ $+\gamma_{0}+\gamma_{2}+\gamma_{3}+\cdots, \alpha_{r}={ }_{d f} \chi_{r}$ for $r \geqq 2$. Then $\alpha_{0}, \alpha_{1}, \cdots$ satisfy the requirements.

The set $\alpha$ is said to be many-one reducible to $\beta$ (denoted by: " $\alpha(m-1)$ red $\beta^{\prime \prime}$ ), if there exists a recursive function $f(n)$ which maps $\alpha$ into $\beta$ and $\alpha^{\prime}$ into $\beta^{\prime}$.

Theorem T 1.3. If $\alpha(m-1) \operatorname{red} \beta$ and $\alpha$ is c.p., then $\beta$ is c.p. 
Proof. Let $f(n)$ be a recursive function such that $f(\alpha) \subset \beta$ and $f\left(\alpha^{\prime}\right) \subset \beta^{\prime}$, $g(n)$ a recursive function such that $\omega_{g(n)}=f^{-1}\left(\omega_{n}\right)$ and $h(n)$ a c.p. function of $\alpha$. Then

$$
h g(n) \in\left(\alpha-\omega_{g(n)}\right)+\left(\omega_{g(n)}-\alpha\right) .
$$

Consequently

$$
f h g(n) \in\left(f(\alpha)-f\left(\omega_{o(n)}\right)\right)+f\left(\omega_{g(n)}\right) \cdot f\left(\alpha^{\prime}\right) .
$$

In view of $f\left(\omega_{g(n)}\right)=\omega_{n} \cdot \rho(f), f(\alpha) \subset \beta$, and $f\left(\alpha^{\prime}\right) \subset \beta^{\prime}$, we conclude $f h g(n)$ $\in\left(\beta-\omega_{n}\right)+\left(\omega_{n}-\beta\right)$. Thus $\beta$ is c.p. relative to $f h g(n)$.

\section{Productive sets}

Definition. The set $\alpha$ is productive, if there exists a partial recursive function $p(n)$ such that for all $n$,

$$
\omega_{n} \subset \alpha \rightarrow\left\{\begin{array}{l}
(1) p(n) \text { is defined, } \\
(2) p(n) \in \alpha-\omega_{n} .
\end{array}\right.
$$

If $p(n)$ is a partial recursive function related to $\alpha$ by (1), we say that $\alpha$ is productive relative to $p(n)$ and that $p(n)$ is a productive function of $\alpha$. A productive set is called productive in the sense of Post ( $P$-productive), if at least one of its productive functions is recursive.

REMARKS. (1) Every completely productive set is productive, because $\omega_{n}$ $C \alpha$ implies $\left(\alpha-\omega_{n}\right)+\left(\omega_{n}-\alpha\right)=\alpha-\omega_{n}$. We do not know whether there exists a productive set which is not completely productive.

(2) The reasons that we do not insist that the partial recursive function $p(n)$ mentioned in (1) be recursive are the following. First of all we observe that the values of $p(n)$ for numbers $n$ such that $\sim\left(\omega_{n} \subset \alpha\right)$ have no particular significance for $\alpha$. Secondly, there are cases in which one can prove the existence of a partial recursive function $p(n)$ related to a certain set $\alpha$ by (1) by means of a procedure which breaks down for some values of $n$ such that $\sim\left(\omega_{n} \subset \alpha\right)$. This, of course, does not exclude the possibility that this function $p(n)$ is potentially recursive (i.e., has a recursive extension) or that, while $p(n)$ is not potentially recursive, $\alpha$ has also a recursive productive function.

(3) Every $P$-productive set is productive. The preceding remark suggests the problem whether the converse is true. We have been able to establish only a special case of the converse.

Theorem T 2.1. Every productive set with a r.e. complement is P-productive $\left({ }^{2}\right)$.

Proof. Let $\alpha$ be productive relative to $p(n)$ and let $\alpha^{\prime}=\omega_{k}$. Suppose $i(n)$

(2) It follows from this theorem that the distinction between creative sets and $P$-creative sets as made in [2] is unnecessary. For a set is creative if and only if it is $P$-creative. 
is a recursive function such that $\omega_{i(n)}=\omega_{n} \cdot \omega_{k}$. Let $n$ be any number. We now perform the steps of the sequence

$$
p\langle n ; 0\rangle, g_{i(n)}\langle 0 ; 0\rangle, p\langle n ; 1\rangle, g_{i(n)}\langle 0 ; 1\rangle, p\langle n ; 2\rangle, g_{i(n)}\langle 1 ; 0\rangle, \cdots
$$

until we have reached the first successful step; let $q(n)$ be the value produced by this step. If $\omega_{n} \subset \alpha$ there exists a successful step which produces $p(n)$, while $g_{i(n)}\langle r ; s\rangle$ is unsuccessful for every two numbers $r$ and $s$, since $\omega_{i(n)}=o$. Consequently $\omega_{n} \subset \alpha$ implies $q(n)=p(n) \in \alpha-\omega_{n}$. Now suppose $p(n)$ is not defined for $n=t$. Then $\sim\left(\omega_{t} \subset \alpha\right)$ and $\omega_{i(t)} \neq 0$; this implies that for some numbers $r$ and $s, g_{i(t)}\langle r ; s\rangle$ is the first successful step in the sequence obtained from (2) by replacing $n$ by $t$. Hence $q(t)=g_{i(t)}(r)$. We conclude that $q(n)$ is a recursive productive function of $\alpha$.

THEOREM T 2.2. If $\alpha$ is productive then: (1) $\alpha$ is not r.e., (2) $\alpha$ and $\alpha^{\prime}$ are infinite, (3) $\alpha$ has an infinite r.e. subset.

Proof. If $\alpha$ were r.e., there would exist a number $\in \alpha-\alpha=0$; thus $\alpha$ is not r.e. If $\alpha$ or $\alpha^{\prime}$ were finite, $\alpha$ would be r.e.; hence $\alpha$ and $\alpha^{\prime}$ are infinite. We finally prove that $\alpha$ has an infinite r.e. subset. Let $p(n)$ be a productive function of $\alpha$, then $p(0) \in \alpha$, since $\omega_{0}=o \subset \alpha$. Suppose $b_{0}={ }_{d f} p(0)$; we can now find an index of $\left\{b_{0}\right\}$ and hence an element $\in \alpha-\left\{b_{0}\right\}$, say $b_{1}$. It is now possible to find an index of $\left\{b_{0}, b_{1}\right\}$ and hence an element $\in \alpha-\left\{b_{0}, b_{1}\right\}$, say $b_{2}$. Continuing this procedure we obtain the infinite r.e. subset $\left\{b_{0}, b_{1}, \cdots\right\}$ of $\alpha$.

Proposition A. For any partial recursive function $f(n)$ defined for at least one value of $n$ we can effectively find a recursive function $h(n)$ with the same range as $f(n)$.

Proof. Let the elements of the sequence

$$
f\langle 0 ; 0\rangle, f\langle 0 ; 1\rangle, f\langle 1 ; 0\rangle, \cdots
$$

be denoted by $\Sigma_{0}, \Sigma_{1}, \Sigma_{2}, \cdots$ in the order in which they occur in this sequence. At least one step in $\left\{\Sigma_{n}\right\}$ is successful since $\delta(f) \neq 0$; let $a$ be the element produced by the first successful step in $\left\{\Sigma_{n}\right\}$. Let

$$
h(n)={ }_{d}\left\{\begin{array}{l}
a \text { if } \Sigma_{n} \text { is unsuccessful, } \\
\text { the number produced by } \Sigma_{n} \text { if } \Sigma_{n} \text { is successful. }
\end{array}\right.
$$

Then $h(n)$ is a recursive function with the same range as $f(n)$.

Proposition B. The set $\alpha$ is productive if and only if $\alpha \neq 0$ and there exists $a$ partial recursive function $q(n)$ such that for all $n$,

$$
\left.\begin{array}{l}
\omega_{n} \neq o \\
\omega_{n} \subset \alpha
\end{array}\right\} \rightarrow\left\{\begin{array}{l}
(1) q(n) \text { is defined, } \\
(2) q(n) \in \alpha-\omega_{n} .
\end{array}\right.
$$

Proof. The "only if" part is obvious. Now suppose $\alpha$ and $q(n)$ satisfy the 
hypotheses of the "if" part. Let $a \in \alpha$ and $i(n)$ be a recursive function such that $\omega_{i(n)}=\omega_{n}+\{a\}$. It is now readily verified that $\alpha$ is productive relative to $q i(n)$.

Proposition C. The set $\alpha$ is productive if and only if there exists a partial recursive function $r(n)$ such that for all $n$,

$$
\omega_{n} \subset \alpha \rightarrow\left\{\begin{array}{l}
(1) r(n) \text { is defined, } \\
(2) \omega_{r(n)} \subset \alpha-\omega_{n}, \\
(3) \omega_{r}(n) \notin Q .
\end{array}\right.
$$

Proof. The "if" part being obvious we can restrict our attention to the "only if" part. Let $\alpha$ be productive relative to $p(n)$ and suppose $\beta=\omega_{k}$ is a r.e. subset of $\alpha$. Let $c_{0}=_{d f} p(k)$, then $c_{0} \in \alpha-\beta$. Using $p(n)$ we can now obtain an element $c_{1} \in \alpha-\left(\beta+\left\{c_{0}\right\}\right)$, an element $c_{2} \in \alpha-\left(\beta+\left\{c_{0}, c_{1}\right\}\right)$, etc. In this way we obtain an infinite r.e. subset $\left\{c_{0}, c_{1}, \cdots\right\}$ of $\alpha-\beta$. This completes the proof.

Let $\alpha$ be a productive set and $\Delta$ a general method which enables us to find for any given r.e. subset $\beta$ of $\alpha$ an element $b_{\beta} \in \alpha-\beta$. The element $b_{\beta}$ might be considered as a witness of the fact that $\beta C_{+} \alpha$. The set of all witnesses, i.e., the set $\pi_{\Delta}$ over which $b_{\beta}$ ranges if $\beta$ ranges over the class of all r.e. subsets of $\alpha$, is called the productive center of $\alpha$ with respect to the method $\Delta$. The following questions arise:

(1) Does the fact that $\alpha$ has $\pi$ as a productive center imply that $\pi=\alpha$ ?

(2) If the answer to (1) is negative,

(a) how many productive centers can a productive set have?

(b) does there exist a productive set which is equal to one of its productive centers?

(c) can a productive set have two disjoint productive centers?

Before we can answer these questions it is necessary to give a more formal definition of the concept of a productive center.

Definition. Dom $\alpha={ }_{d f}\left\{n \mid \omega_{n} \subset \alpha\right\}$. Dom $\alpha$ is called the domain of $\alpha$.

Definition. Let $\alpha$ be productive relative to $p(n)$. Then $\pi(\alpha, p)$ $={ }_{d f} p(\operatorname{Dom} \alpha)$. The set $\pi(\alpha, p)$ is called the productive center of $\alpha$ relative to $p(n)$. The subset $\pi$ of $\alpha$ is called a productive center of $\alpha$, if $\pi=\pi(\alpha, p)$ for some productive function $p(n)$ of $\alpha$.

Proposition D. If $\pi_{0}$ is a productive center of the productive set $\alpha$, there exists a productive center $\pi_{1}$ of $\alpha$ such that $\pi_{1} \subset \pi_{0}$ and $\pi_{0}-\pi_{1} \notin Q$.

Proof. Let $p_{0}(n)$ be a productive function of $\alpha$ such that $\pi_{0}=\pi\left(\alpha, p_{0}\right)$. Using $p_{0}(n)$ we can now construct an infinite r.e. subset $\beta$ of $\pi_{0}$ (see the proof of the third part of $T 2.2)$. Let $i(n)$ be a recursive function such that $\omega_{i(n)}$ $=\omega_{n}+\beta$. Suppose $p_{1}(n)=_{d f} p_{0} i(n)$ and $\pi_{1}=_{d f} \pi\left(\alpha, p_{1}\right)$. It is now readily seen 
that $p_{1}(n)$ is a productive function of $\alpha$ and that $\pi_{1} \subset \pi_{0}$. Moreover, $\pi_{0}-\pi_{1} \notin Q$ because $\beta \subset \pi_{0}-\pi_{1}$.

CoRollary. Every productive set $\alpha$ has a productive center $\pi_{1}$ such that $\alpha-\pi_{1}$ is infinite.

THEOREM T 2.3. Every productive set has exactly denumerably many productive centers and exactly denumerably many productive functions.

Proof. A productive set $\alpha$ has infinitely many productive centers by proposition D. Furthermore, $\alpha$ has at most denumerably many productive centers, because each of them is the image of Dom $\alpha$ under some partial recursive function, while there are only denumerably many partial recursive functions. Thus $\alpha$ has exactly denumerably many productive centers. Different productive centers of $\alpha$ are images of Dom $\alpha$ under different productive functions of $\alpha$. Táking into account that $\alpha$ has at most denumerably many productive functions, we conclude that $\alpha$ has exactly denumerably many productive functions.

Theorem T 2.4. Let $\alpha$ be productive relative to $p(n)$ and let $\pi(\alpha, p) \subset \beta \subset \alpha$. Then $\beta$ is productive relative to $p(n)$ and $\pi(\beta, p) \subset \pi(\alpha, p)$.

Proof. Let $\omega_{n} \subset \beta$; this implies $\omega_{n} \subset \alpha$. Then $p(n) \notin \omega_{n}$ since $p(n) \in \alpha-\omega_{n}$, and $p(n) \in \beta$ since $p(n) \in \pi(\alpha, p)$. Thus $p(n) \in \beta-\omega_{n}$. Clearly, $\pi(\beta, p) \subset \pi(\alpha, p)$ since Dom $\beta \subset$ Dom $\alpha$.

CoRollary 1. If $\alpha$ is productive relative to $p(n)$, then $\pi(\alpha, p)$ is also productive relative to $p(n)$.

COROLlaRY 2. Every productive set includes exactly c productive sets.

Proof. It suffices to show that every productive set includes at least $c$ productive sets. Let $\alpha$ be productive, then $\alpha$ has a productive center $\pi$ such that $\alpha-\pi$ is infinite by the corollary of Proposition D. Suppose $\tau \subset \alpha-\pi$. Then $\pi \subset \pi+\tau \subset \alpha$, different choices of $\tau$ yield different sets $\pi+\tau$, and $\tau$ can be chosen in $c$ different ways. Hence $\alpha$ includes at least $c$ productive sets.

TheOREM T $2.5\left(^{3}\right)$. Let $\alpha$ be a productive set with a productive center $\pi$ and let $\beta$ be r.e. Then:

(1) $\beta \subset \alpha$ implies that $\alpha-\beta$ is productive with a productive center included in $\pi$,

(2) $\alpha \subset \beta$ implies that $\alpha+\beta^{\prime}$ is productive with a productive center included in $\pi$.

Proof. Let $p(n)$ be a productive function of $\alpha$ such that $\pi=\pi(\alpha, p)$.

(1) Let $\gamma$ be a r.e. subset of $\alpha-\beta$. Then $\beta+\gamma$ is a r.e. subset of $\alpha$ and we

(3) T 2.5 was suggested by $[4$, p. 296, lines 6-8]. The present theorem is, however, more general, since it applies to all productive sets, not only to those with a r.e. complement. 
can find an element $a \in \alpha-(\beta+\gamma)=(\alpha-\beta)-\gamma$. The element $a$ belongs to $\pi$ if we use $p(n)$ to find it.

(2) Let $\delta$ be a r.e. subset of $\alpha+\beta^{\prime}$. Since $\delta \subset \alpha+\beta^{\prime}$ implies $\beta \delta \subset \beta \alpha$, it follows that $\beta \delta$ is a r.e. subset of $\alpha$. We can now find an element $a \in \alpha-\beta \delta$. In view of the fact that $\alpha \subset \beta$ implies $\alpha \beta^{\prime}=0$, we see that $\alpha-\beta \delta=\alpha-\delta$. But $\alpha-\delta$ $C\left(\alpha+\beta^{\prime}\right)-\delta$. Thus $a \in\left(\alpha+\beta^{\prime}\right)-\delta$. The element $a$ belongs to $\pi$ if we use $p(n)$ to find it.

TheOREM T 2.6. (1) A productive set intersects a simple set in a productive set.

(2) A creative set intersects a simple set in a creative set.

Proof. (1) Let $\alpha$ be productive, $\zeta$ simple, and $\beta$ a r.e. subset of $\alpha \zeta$. By Proposition $\mathrm{C}$ we can find an infinite r.e. subset $\gamma$ of $\alpha-\beta$. The set $\zeta^{\prime}$ being immune, $\gamma \zeta$ must be nonempty; we can now find an element in $\gamma \zeta$, say $k$. It follows from $k \in \gamma \zeta$ and $\gamma \subset \alpha-\beta$ that $k \in \alpha \zeta-\beta$.

(2) Let $\alpha$ be creative and $\zeta$ simple. Then $\alpha \zeta$ is r.e. and all we have to prove is that $(\alpha \zeta)^{\prime}$ is productive. We know that $\alpha^{\prime} \zeta$ is productive, because $\alpha^{\prime}$ is productive and $\zeta$ simple. Furthermore, $\alpha^{\prime} \zeta \subset \zeta$, where $\zeta$ is r.e. Thus $\alpha^{\prime} \zeta+\zeta^{\prime}$ is productive by the second part of T 2.5. But $\alpha^{\prime} \zeta+\zeta^{\prime}=(\alpha \zeta)^{\prime}$. Hence $(\alpha \zeta)^{\prime}$ is productive.

Definition. Let $\alpha$ be productive. Then Prod $\alpha=_{d f}\left\{n \mid g_{n}(x)\right.$ is a productive function of $\alpha$.

Theorem T 2.7. Let $\alpha$ be productive. Then Dom $\alpha$ and Prod $\alpha$ are two disjoint productive sets.

Proof. Let $p(n)$ be a productive function of $\alpha$ and $\beta$ a nonempty r.e. subset of Dom $\alpha$. By Proposition A we can find a recursive function ranging over $\beta$, say $b(n)$. Numbers $k$ and $l$ can now be found such that $\omega_{k}=\omega_{b(0)}+\omega_{b(1)}+\ldots$ and $\omega_{l}=\{p(k)\}$. The fact that $\omega_{k} \subset \alpha$ implies $p(k) \in \alpha-\omega_{k}$; hence $l \in \operatorname{Dom} \alpha$ $-\beta$. It follows by Proposition B that Dom $\alpha$ is productive. We proceed to show that Prod $\alpha$ is productive. Let $\beta$ be a nonempty r.e. subset of Prod $\alpha$, then we can find a recursive function ranging over $\beta$, say $b(t)$. We shall now define a productive function $g_{k}(n)$ of $\alpha$ which is different from $g_{b(t)}(n)$ for every $t$. Let $\omega_{n} \subset \alpha$, then $g_{b(t)}(n) \in \alpha-\omega_{n}$ for every $t$. Put $\gamma_{n}=g_{d f}\left\{g_{b(0)}(n)\right.$, $\left.g_{b(1)}(n), \cdots\right\}$, then $\gamma_{n}$ and $\gamma_{n}+\omega_{n}$ are r.e.; moreover $\gamma_{n}+\omega_{n} \subset \alpha$. We can now find a partial recursive function $i(n)$ such that $\omega_{i(n)}=\omega_{n}+\gamma_{n}$ for $n$ $\in \operatorname{Dom} \alpha$. Let $q(n)$ be any productive function of $\alpha$. Then $q i(n) \in \alpha-\left(\omega_{n}+\gamma_{n}\right)$ for $n \in \operatorname{Dom} \alpha$. This implies in the first place that $q i(n) \in \alpha-\omega_{n}$ for $n \in \operatorname{Dom} \alpha$, i.e., that $q i(n)$ is a productive function of $\alpha$, and in the second place that $q i(n) \notin \gamma_{n}$ for $n \in \operatorname{Dom} \alpha$, i.e., that $q i(n)$ is different from $g_{b(t)}(n)$ for every $t$. We can find a number $k$ such that $q i(n)=g_{k}(n)$. Hence $k \in \operatorname{Prod} \alpha-\beta$. We conclude that Prod $\alpha$ is productive. Suppose Dom $\alpha$ and Prod $\alpha$ had an element in common, say $s$. Then $\omega_{s} \subset \alpha$ and $g_{s}(n)$ is a productive function of 
$\alpha$. This leads to a contradiction, since we would simultaneously have $g_{s}(s)$ $\in \alpha-\omega_{s}$ and $g_{s}(s) \in \rho\left(g_{s}(n)\right)=\omega_{s}$. Thus Dom $\alpha$ and Prod $\alpha$ are disjoint.

Corollary. Any productive function of a productive set $\alpha$ is defined for infinitely many numbers which do not belong to Dom $\alpha$.

Proof. Let $\alpha$ be productive relative to $p(n)$. Then Dom $\alpha \subset \delta(p)$. But Dom $\alpha$ is productive and $\delta(p)$ is r.e. Furthermore, every set obtained by omitting a finite number of elements from $\delta(p)$ is also r.e.

Theorem T 2.8. (1) If $\alpha(m-1)$ red $\beta$ and $\alpha$ is productive, then $\beta$ is productive.

(2) If $\alpha(m-1)$ red $\beta$ and $\alpha$ is creative, then $\beta$ is creative.

Proof. See [2, Theorems 2.1 and 2.2] $\left({ }^{4}\right)$.

We shall now discuss two productive sets among whose productive centers denumerably many are mutually disjoint.

Definition. The number $n$ is called an index of the partial recursive function $f(x)$ if $f(x)=g_{n}(x)$.

THEOREM T 2.9. Let $\alpha$ be the set of all indices of recursive functions, let $p \neq q$, and let $\pi_{p q}$ be the set of all indices of recursive functions whose range is included in $\{p, q\}$. Then $\alpha$ is a productive set with a productive center included in $\pi_{p q}$. Among the productive centers of $\alpha$ denumerably many are mutually disjoint.

Proof. Let $\beta$ be a nonempty r.e. subset of $\alpha$, then we can find a recursive function ranging over $\beta$, say $b(n)$. Suppose $\Gamma$ is the collection of all recursive functions and

$$
\Delta_{\beta}={ }_{d f}\left\{g_{b(0)}(x), g_{b(1)}(x), \cdots\right\}
$$

Put

$$
c_{p q}(x)=d_{d f}\left\{\begin{array}{lll}
p & \text { if } & g_{b(x)}(x) \neq p \\
q & \text { if } & g_{b(x)}(x)=p
\end{array}\right.
$$

then $c_{p q}(x) \in \Gamma-\Delta_{\beta}$. We can now find a number $k$ such that $g_{k}(x)=c_{p q}(x)$. It follows that $k \in \alpha-\beta$ and $k \in \pi_{p q}$. Hence $\alpha$ is a productive set with a productive center included in $\pi_{p q}$. Since each of the sets $\pi_{01}, \pi_{23}, \pi_{45}, \cdots$ includes a productive center of $\alpha$, we conclude that among the productive centers of $\alpha$ denumerably many are mutually disjoint.

Using T 2.4 it follows that any set $\beta$ with the property that $\pi_{p q} \subset \beta \subset \alpha$

(4) In the proof of Theorem 2.1 [2, p. 499] the word "partial" should be omitted from line 4 from the foot of p. 499 and inserted before the word "recursive" in line 2 from the foot of p. 499. 
for two different numbers $p$ and $q$ is also productive; this enables us to prove the productivity of a great variety of sets of indices of recursive functions.

Definition. Let $\alpha$ be an infinite r.e. set. Then $\bar{\theta}(\alpha)={ }_{d f}$ the set of all indices of 1-1 recursive functions ranging over $\alpha$.

THEOREM T 2.10. Let $\alpha$ be an infinite r.e. set. Then $\bar{\theta}(\alpha)$ is a productive set among whose productive centers denumerably many are mutually disjoint.

Proof. Let $a(x)$ be a 1-1 recursive function ranging over $\alpha$; let $\beta$ be a nonempty r.e. subset of $\bar{\theta}(\alpha)$ and $b(n)$ a recursive function ranging over $\beta$. Suppose $f(n, x)={ }_{d f} g_{b(n)}(x)$. We now transform $a(x)$ into a function $a^{*}(x)$ in the following way. Leave $a(0)$ as it is, i.e., let $a^{*}(0)={ }_{d f} a(0)$. Compare the ordered pairs $(a(1), a(2))$ and $(f(0,1), f(0,2))$; if they are different, leave $a(1)$ and $a(2)$ alone, otherwise interchange them; thus, in the latter case: $a^{*}(1)={ }_{d f} a(2)$, $a^{*}(2)={ }_{d f} a(1)$. Now compare $(a(3), a(4))$ with $(f(1,3), f(1,4))$ and proceed likewise. Continuing this procedure we obtain the function $a^{*}(x)$ with the five properties: $\rho\left(a^{*}\right)=\alpha, a^{*}(0)=a(0), a^{*}(x)$ is 1-1 and recursive, $a^{*}(x)$ $\neq f(n, x)$ for every $n$. Thus, if $i$ is an index of $a^{*}(x)$ then $i \in \bar{\theta}(\alpha)-\beta$. Observe that all indices $i$ obtained in this way for different choices of $\beta$ are such that $g_{i}(0)=a(0)$. If we would start instead of with $a(n)$ with a 1-1 recursive function $a_{1}(n)$ ranging over $\alpha$ such that $a_{1}(0) \neq a(0)$, the corresponding number $i$ would range over a productive center disjoint from that corresponding to the construction based on $a(n)$. Since $a_{1}(0)$ can have denumerably many different values, it follows that among the productive centers of $\bar{\theta}(\alpha)$ denumerably many are mutually disjoint.

Theorems T 2.9 and T 2.10 imply that given any effective process for identifying partial recursive functions as recursive (or as 1-1 recursive with a given range) we can effectively find a recursive function (respectively, a 1-1 recursive function with the given range) which the process fails to identify as such.

A member of a class of sets is called minimal if it does not properly include any other member of that class. The class of all productive sets has no minimal member; for every productive set includes $c$ productive sets by the corollary of $\mathrm{T} 2.4$.

Definition. The partial recursive function $p(n)$ is called productive if it is a productive function of at least one productive set.

Theorem T 2.11. Let $p(n)$ be a productive function. A mong all sets productive relative to $p(n)$ there is a smallest one; this smallest set is its own productive center relative to $p(n)$.

Proof. Let $p(n)$ be a productive function and $\alpha$ the intersection of all sets productive relative to $p(n)$. It is now easy to show that $\alpha$ itself is productive relative to $p(n)$; hence $\alpha$ is the smallest set productive relative to $p(n)$. By Corollary 1 of $\mathrm{T} 2.4, \pi(\alpha, p)$ is a subset of $\alpha$ which is productive relative to 
$p(n)$. Thus $\pi(\alpha, p)=\alpha$.

Definition. A set productive relative to $p(n)$ is called minimal relative to $p(n)$ if it is the smallest set productive relative to $p(n)$. A productive set is called minimal if it is minimal relative to at least one of its productive functions.

We mention the following properties of minimal productive sets. First of all, among the minimal productive sets denumerably many are mutually disjoint; this follows from $\mathrm{T} 2.9$, the fact that every productive center of a productive set is productive, and the fact that every productive set includes a minimal productive set. Secondly, it is easily seen that every productive set includes infinitely many minimal productive sets. For let $\alpha$ be productive; then $\alpha$ includes a minimal productive set, say $\mu_{0}$. By the corollary of Proposition $\mathrm{D}, \mu_{0}$ has a productive center $\pi_{1}$ such that $\mu_{0}-\pi_{1}$ is infinite. Then $\pi_{1}$ includes a minimal productive set $\mu_{1}$ and $\mu_{1} \neq \mu_{0}$. Since this procedure can be continued indefinitely, it follows that $\alpha$ includes infinitely many minimal productive sets. Finally we observe that, while there exist $c$ productive sets, there are only denumerably many minimal ones among them. For every minimal productive set is completely determined by some productive function and there are only denumerably many productive functions.

The proof of $\mathrm{T} 2.11$ was only an existence proof. The following proof is in a certain sense more constructive, though it uses transfinite induction.

Second proof of T 2.11. In the following the letters " $\nu$," " $\phi$," " $\psi$ " will denote ordinals in the first or second number class. Let $p(n)$ be a productive function. We define

$$
\mu_{0}(p)=_{d f} p(\text { Dom } o), \quad \mu_{\phi}(p)={ }_{d f} p\left(\operatorname{Dom} \sum_{p<\phi} \mu_{\nu}(p)\right), \quad \text { for } \quad \phi>0 .
$$

Clearly, $\sum_{\nu<\phi} \mu_{\nu}(p) \subset \mu_{\phi}(p)$ for all $\phi$. We claim that there exists an ordinal $\psi>0$ in the first or second numberclass such that $\sum_{\nu<\psi} \mu_{\nu}(p)=\mu_{\psi}(p)$. For consider the transfinite sequence $\left\{\mu_{\phi}(p)-\sum_{\nu<\phi} \mu_{\nu}(p)\right\}$ whose first element is $\mu_{1}(p)-\mu_{0}(p)$. The elements of this sequence are mutually disjoint subsets of $\epsilon$. But $\epsilon$ is denumerable and the sequence has nondenumerably many elements. Thus at most denumerably many elements in the sequence can be nonempty and among the ordinals $\phi$ such that $\mu_{\phi}(p)-\sum_{\nu<\phi} \mu_{\nu}(p)=0$ and $\phi>0$ there must be a first one, say $\psi$. Hence $\sum_{\nu<\psi} \mu_{\nu}(p)=\mu_{\psi}(p)$. We now define $\mu(p)=_{d f} \mu_{\psi}(p)$ and proceed to show that $\mu(p)$ is the minimal productive set relative to $p(n)$. Let $\alpha$ be productive relative to $p(n)$. Then $o \subset \alpha$; moreover, $\beta \subset \alpha$ implies $p(\operatorname{Dom} \beta) \subset \alpha$. Hence $\sum_{\phi} \mu_{\phi}(p) \subset \alpha$, i.e., $\mu(p) \subset \alpha$. To complete the proof it suffices to show that $\mu(p)$ itself is productive relative to $p(n)$. Let $\omega_{n} \subset \mu(p)$; then $p(n) \notin \omega_{n}$ because $\omega_{n} \subset \alpha$; furthermore $p(n) \in \mu(p)$ by the definition of $\mu(p)$. Hence $p(n) \in \mu(p)-\omega_{n}$.

It follows from this proof that if $p(n)$ is a productive function, the minimal productive set relative to $p(n)$ can be defined as the smallest set $\sigma$ (or the intersection of all sets $\sigma)$ such that $p(\operatorname{Dom} \sigma) \subset \sigma$. 


\section{Contraproductive SETS}

Definition. The set $\alpha$ is contraproductive, if there exists a partial recursive function $p(n)$ such that for all $n$,

$$
\alpha \subset \omega_{n} \rightarrow \begin{cases}(1) & p(n) \text { is defined, } \\ (2) & p(n) \in \omega_{n}-\alpha .\end{cases}
$$

If $p(n)$ is a partial recursive function related to $\alpha$ by (1), we say that $\alpha$ is contraproductive relative to $p(n)$ and that $p(n)$ is a contraproductive function of $\alpha$.

REMARKS. (1) Every completely productive set is contraproductive, since $\alpha \subset \omega_{n}$ implies $\left(\alpha-\omega_{n}\right)+\left(\omega_{n}-\alpha\right)=\omega_{n}-\alpha$. We shall prove (T 3.3) that there exist contraproductive sets which are not productive (hence not completely productive), but we do not know whether there exist productive sets which are not contraproductive.

(2) There exist exactly $c$ contraproductive sets, because there exist exactly $c$ completely productive sets.

THEOREM T 3.1. If $\alpha$ is contraproductive then (1) $\alpha$ is not r.e., (2) $\alpha$ and $\alpha^{\prime}$ are infinite, (3) $\alpha^{\prime}$ has an infinite r.e. subset.

Proof. The first two parts are obvious. The third part can be proved similarly to the third part of T 2.2 using the fact that $\alpha \subset \omega_{1}=\epsilon$.

The following proposition is easily verified (cf. Proposition $\mathrm{C}$ in $\S \mathrm{II}$ ).

Proposition E. The set $\alpha$ is contraproductive if and only if there exists a partial recursive function $r(n)$ such that for all $n$,

$$
\alpha \subset \omega_{n} \rightarrow\left\{\begin{array}{l}
(1) r(n) \text { is defined, } \\
(2) \omega_{r(n)} \subset \omega_{n}-\alpha, \\
(3) \omega_{r(n)} \notin Q .
\end{array}\right.
$$

Definition. Codom $\alpha={ }_{d f}\left\{n \mid \alpha \subset \omega_{n}\right\}$.

Definition. Let $\alpha$ be contraproductive relative to $p(n)$. Then $\pi_{c}(\alpha, p)$ $={ }_{d f} p($ Codom $\alpha)$. The set $\pi_{c}(\alpha, p)$ is called the contraproductive center of $\alpha$ relative to $p(n)$. The subset $\pi$ of $\alpha^{\prime}$ is called a contraproductive center of $\alpha$, if $\pi=\pi_{c}(\alpha, p)$ for some contraproductive function $p(n)$ of $\alpha$.

The following three statements are proved similarly to Proposition D, its corollary, and T 2.3.

PROPOSITION F. If $\pi_{0}$ is a contraproductive center of a contraproductive set $\alpha$, there exists a contraproductive center $\pi_{1}$ of $\alpha$ such that $\pi_{1} C_{+} \pi_{0}$ and $\pi_{0}-\pi_{1} \notin Q$.

COROLlaRY. Every contraproductive set $\alpha$ has a contraproductive center $\pi_{1}$ such that $\alpha^{\prime}-\pi_{1}$ is infinite.

THEOREM T 3.2. Every contraproductive set has exactly denumerably many 
contraproductive centers and exactly denumerably many contraproductive functions.

THEOREM T 3.3. (1) Every set $\alpha$ such that $\alpha^{\prime} \in F-E$ is contraproductive and has $\alpha^{\prime}$ as one of its contraproductive centers.

(2) Let $\alpha^{\prime}$ be a set of $F-E$ which is not creative. Then $\alpha$ is contraproductive, but not productive.

Proof. (1) Let $\beta=\alpha^{\prime}$ and suppose $\beta \in F-E$. Let $i(n)$ be a recursive function such that $\omega_{i(n)}=\omega_{n} \cdot \alpha^{\prime}$. Then $\omega_{i(n)}=\omega_{n}-\alpha(1)$. There exists a partial recursive function $p(n)$ such that $\omega_{n} \neq o \rightarrow p(n) \in \omega_{n}$ (2). Note that $\alpha \subset \omega_{n}$ implies $\omega_{n} \neq 0$. It now follows from (1) and (2) that $\alpha$ is contraproductive relative to $p i(n)$. Suppose $b(n)$ is a $1-1$ recursive function ranging over $\beta, \delta$ an infinite recursive set of indices of $\epsilon$, and $d(n)$ a 1-1 recursive function ranging over $\delta$. We now define a partial recursive function $r(n)$ by the following three conditions:

(a) $r(n)$ is defined if and only if $p i(n)$ is,

(b) if $n \in \delta$ and $k=d^{-1}(n), r(n)=b(k)$,

(c) if $n \notin \delta, r(n)=p i(n)$.

We conclude that $r(n)$ is a contraproductive function of $\alpha$ such that $\beta$ $C r(\operatorname{Codom} \alpha)$. Thus $\pi_{c}(\alpha, r)=\beta$.

(2) Let $\alpha^{\prime}$ satisfy the hypotheses. Then $\alpha$ is contraproductive because $\alpha^{\prime} \in F-E$, and $\alpha$ is not productive, because $\alpha^{\prime}$ is not creative.

The following theorem and its two corollaries are the contraproductive analogues of T 2.4 and its corollaries; they are readily verified.

THEOREM T 3.4. Let $\alpha$ be contraproductive relative to $p(n)$ and let $\alpha \subset \beta$ $\subset \pi_{c}^{\prime}(\alpha, p)$. Then $\beta$ is contraproductive relative to $p(n)$ and $\pi_{c}(\beta, p) \subset \pi_{c}(\alpha, p)$.

CoROLlaRY 1. If $\alpha$ is contraproductive relative to $p(n)$, then $\pi_{c}^{\prime}(\alpha, p)$ is also contraproductive relative to $p(n)$.

COROLLARY 2. Every contraproductive set is included in exactly c contraproductive sets.

THEOREM T 3.5. Let $\alpha$ be a contraproductive set with a contraproductive center $\pi$ and let $\beta$ be r.e. Then $\alpha \subset \beta$ implies that $\alpha+\beta^{\prime}$ is contraproductive with a contraproductive center included in $\pi$.

Proof. Let $p(n)$ be a contraproductive function of $\alpha$ such that $\pi=\pi_{c}(\alpha, p)$. Suppose $\alpha+\beta^{\prime} \subset \gamma$, where $\gamma$ is r.e. Then $\left(\alpha+\beta^{\prime}\right) \beta \subset \beta \gamma$. But $\left(\alpha+\beta^{\prime}\right) \beta=\alpha$, since $\alpha \subset \beta$. Hence $\alpha \subset \beta \gamma$, where $\beta \gamma$ is r.e. We can now find a number $k \in \beta \gamma-\alpha$. Note that $\beta \gamma-\alpha=\gamma-\left(\alpha+\beta^{\prime}\right)$. Hence $\alpha+\beta^{\prime}$ is contraproductive. The element $k$ belongs to $\pi$, if we use $p(n)$ to find it.

TheOREM T 3.6. If $\alpha(m-1)$ red $\beta$ and $\alpha$ is contraproductive, then $\beta$ is contraproductive. 
Proof. Let $f(n)$ be a recursive function such that $f(\alpha) \subset \beta$ and $f\left(\alpha^{\prime}\right) \subset \beta^{\prime}$. There exists a recursive function $g(n)$ such that $\omega_{g(n)}=f^{-1}\left(\omega_{n}\right)$. Let $h(n)$ be a contraproductive function of $\alpha$. Then $\beta \subset \omega_{n} \rightarrow f^{-1}(\beta) \subset f^{-1}\left(\omega_{n}\right) \rightarrow \alpha \subset \omega_{o(n)}$ $\rightarrow h g(n) \in \omega_{o(n)}-\alpha \rightarrow f h g(n) \in f\left(\omega_{o(n)}\right) \cdot f\left(\alpha^{\prime}\right) \rightarrow f h g(n) \in \omega_{n}-\beta$. We conclude that $\beta$ is contraproductive relative to $f h g(n)$.

TheOREM T 3.7. Among all sets contraproductive relative to $p(n)$ there is a largest one; this largest set has its complement as its contraproductive center relative to $p(n)$.

Proof. Both proofs of $\mathrm{T} 2.11$ discussed in $\S I I$ can be easily modified to prove the present theorem.

The concepts contraproductive function, maximal contraproductive set relative to $p(n)$, and maximal contraproductive set can be defined in an obvious manner. There are denumerably many maximal contraproductive sets and the contraproductive set maximal relative to the contraproductive function $p(n)$ can be defined as the largest set $\sigma$ (or the union of all sets $\sigma$ ) such that, for all $n, \sigma \subset \omega_{n} \rightarrow p(n) \in \omega_{n}$.

\section{Semiproductive SETS}

Definition. The set $\alpha$ is semiproductive if there exists a partial recursive function $p(n)$ such that for all $n$,

$$
\omega_{n} \subset \alpha \rightarrow\left\{\begin{array}{l}
(1) p(n) \text { is defined, } \\
(2) \omega_{n} \subset_{+} \omega_{p(n)} \subset \alpha .
\end{array}\right.
$$

If $p(n)$ is a partial recursive function related to $\alpha$ by (1), we say that $\alpha$ is semiproductive relative to $p(n)$ and that $p(n)$ is a semiproductive function of $\alpha$.

REMARKs (1) Every productive set is semiproductive. For let $\alpha$ be productive relative to $p(n)$; then $\alpha$ is semiproductive relative to any partial recursive function $q(n)$ such that $\omega_{q(n)}=\omega_{n}+\{p(n)\}$ for $n \in$ Dom $\alpha$. We shall find ( $T$ 4.2) that there exist semiproductive sets which are neither productive nor contraproductive.

(2) There are exactly $c$ semiproductive sets, since there are exactly $c$ productive sets.

The following theorem can be established by a slight modification of the proof of $\mathrm{T} 2.2$.

Theorem T 4.1. If $\alpha$ is semiproductive, then (1) $\alpha$ is not r.e., (2) $\alpha$ and $\alpha^{\prime}$ are infinite, (3) $\alpha$ has an infinite r.e. subset.

Proposition B of $\S$ II remains valid if we replace "productive" by "semiproductive" and " $q(n) \in \alpha-\omega_{n}$ " by " $\omega_{n} \subset_{+} \omega_{q(n)} \subset \alpha$."

Proposition G. The set $\alpha$ is semiproductive if and only if there exists a partial recursive function $t(n)$ such that for all $n$, 


$$
\omega_{n} \subset \alpha \rightarrow \begin{cases}(1) & t(n) \text { is defined } \\ \text { (2) } & \omega_{t(n)} \subset \alpha, \\ (3) & \omega_{t(n)}-\omega_{n} \neq 0 .\end{cases}
$$

Proof. The "only if" part is obvious. Under the hypotheses of the "if" part there exists a partial recursive function $p(n)$ such that for all $n, \omega_{p(n)}$ $=\omega_{n}+\omega_{t(n)}$. Then $\alpha$ is semiproductive relative to $p(n)$.

Proposition H. The set $\alpha$ is semiproductive if and only if there exists a partial recursive function $r(n)$ such that for all $n$,

$$
\omega_{n} \subset \alpha \rightarrow \begin{cases}(1) & r(n) \text { is defined, } \\ (2) & \omega_{n} C_{+} \omega_{r(n)} \subset \alpha, \\ (3) & \omega_{r(n)}-\omega_{n} \notin Q .\end{cases}
$$

Proof. The "if" part is obvious. Let $\alpha$ be semiproductive relative to $p(n)$. Then $\omega_{n} \subset \alpha$ implies:

$$
\omega_{n} \subset_{+} \omega_{p(n)} \subset_{+} \omega_{p p(n)} \subset_{+} \cdots \subset \alpha .
$$

Thus any partial recursive function $r(n)$ such that $\omega_{r(n)}=\omega_{p(n)}+\omega_{p p(n)}+\cdots$ for $n \in$ Dom $\alpha$ satisfies the requirements.

Theorem $T$ 4.2. There exists a semiproductive set which is neither productive nor contraproductive.

Proof. Let $j_{n}(x)=_{d f} j(n, x)$ and $\eta_{n}={ }_{d f} \rho\left(j_{n}(x)\right)$, then $\eta_{0}+\eta_{1}+\ldots$ is a decomposition of $\epsilon$ into mutually disjoint infinite recursive sets. Suppose $\zeta$ is a simple set and $i_{n}$ a strictly increasing function ranging over $\zeta^{\prime}$. Observe that $i_{n} \geqq n$ and that $i_{n}$ ranges over an immune set. We define:

$$
\gamma={ }_{d f}\left\{j_{n}\left(i_{n}\right) \mid \eta_{n} \subset \omega_{n}\right\}
$$

It follows that $\gamma$ is a subset of $\left\{j_{0}\left(i_{0}\right), j_{1}\left(i_{1}\right), \cdots\right\}$ and that $j_{n}\left(i_{n}\right) \in \gamma$ $\leftrightarrow \eta_{n} \subset \omega_{n}$. Thus we see that for every $n, \eta_{n}$ contributes either no element to $\gamma$ or exactly one element, namely $j_{n}\left(i_{n}\right)$. The fact that $i_{n} \geqq n \mathrm{im}$ plies that $j_{n}\left(i_{n}\right)$ does not occur in $\left\{j_{n}(0), \cdots, j_{n}(n-1)\right\}$; all elements of the matrix $\left\|j_{n}(x)\right\|$ which occur under the principal diagonal belong therefore to $\gamma^{\prime}$. Let

$$
\alpha={ }_{d f} \sum_{i=1}^{\infty}\left\{j_{i}(0), \cdots, j_{i}(i-1)\right\},
$$

then $\alpha$ is r.e. and $\alpha \subset \gamma^{\prime}$. We shall now prove: (a) $\gamma^{\prime}$ is semiproductive, (b) $\gamma^{\prime}$ is not productive, (c) $\gamma^{\prime}$ is not contraproductive.

Proof of (a). Let $\omega_{n} \subset \gamma^{\prime}$; then $\omega_{n} \cdot \gamma=0$; it is therefore impossible that $\eta_{n} \subset \omega_{n}$, for this would imply $\eta_{n} \cdot \gamma \neq 0$, hence also $\omega_{n} \cdot \gamma \neq 0$. Thus $\sim\left(\eta_{n} \subset \omega_{n}\right)$ 
and $\eta_{n}-\omega_{n} \neq 0$. It follows from $\sim\left(\eta_{n} \subset \omega_{n}\right)$ and the definition of $\gamma$ that $\eta_{n} \subset \gamma^{\prime}$. Hence $\omega_{n} \subset \gamma^{\prime}$ implies both $\eta_{n} \subset \gamma^{\prime}$ and $\eta_{n}-\omega_{n} \neq 0$. We conclude by Proposition $\mathrm{G}$ that $\boldsymbol{\gamma}^{\prime}$ is semiproductive.

Proof of (b). Let

$$
\beta={ }_{d f} \sum_{n=0}^{\infty} j_{n}(\zeta)
$$

Then $\beta$ is r.e. and $\beta C \gamma^{\prime}$. Recall that $\alpha$ is also r.e.; thus there exists a $k$ such that $\alpha+\beta=\omega_{k} \subset \gamma^{\prime}$. Now suppose $\gamma^{\prime}$ were productive. Then we could find an infinite r.e. set $\left\{p_{0}, p_{1}, \cdots\right\}$ included in $\gamma^{\prime}-\omega_{k}$ by Proposition C. We now locate $p_{0}, p_{1}, \cdots$ in the matrix $\left\|j_{n}(x)\right\|$; let $p_{m}=j_{l(m)}\left(r_{m}\right)$, then $l(m)$ and $r_{m}$ are recursive functions of $m$. The fact that $p_{m} \notin \beta$ implies $p_{m}=j_{l(m)}\left(r_{m}\right)$ $\notin j_{l(m)}(\zeta)$, hence $r_{m} \notin \zeta$. Thus $r_{m} \in \zeta^{\prime}$ for every $m$. Since $\left\{p_{0}, p_{1}, \cdots\right\} \subset \gamma^{\prime}-\alpha$, at most finitely many elements among $p_{0}, p_{1}, \cdots$ can occur in the same column of $\left\|j_{n}(x)\right\|$. It follows that $r_{m}$ has an infinite range, say $\rho$. We now have a contradiction, because $\rho$ is an infinite r.e. subset of the immune set $\zeta^{\prime}$. Hence $\gamma^{\prime}$ is not productive.

Proof of (c). The set $\gamma$ is infinite, because $\gamma^{\prime}$ is semiproductive; furthermore, if $\gamma$ had an infinite r.e. subset $\beta$ we could by locating the elements of $\beta$ in $\left\|j_{n}(x)\right\|$ obtain an infinite r.e. subset of $\zeta^{\prime}$. Thus $\gamma$ has no infinite r.e. subset. It follows that $\gamma$, i.e., the complement of $\gamma^{\prime}$, is immune. But the complement of a contraproductive set includes an infinite r.e. set by $\mathrm{T}$ 3.1. We conclude that $\gamma^{\prime}$ is not contraproductive.

Definition. Let $\alpha$ be semiproductive relative to $p(n)$. Then

$$
\pi_{s}(\alpha, p)=_{d f} \sum_{n \in \text { Dom } \alpha}\left(\omega_{p(n)}-\omega_{n}\right) .
$$

The set $\pi_{s}(\alpha, p)$ is called the semiproductive center of $\alpha$ relative to $p(n)$. The subset $\pi$ of $\alpha$ is called a semiproductive center of $\alpha$, if $\pi=\pi_{s}(\alpha, p)$ for some semiproductive function $p(n)$ of $\alpha$.

REMARK. If $\pi$ is a productive center of the productive set $\alpha, \pi$ is also a semiproductive center of $\alpha$. For suppose $\alpha$ is productive relative to $p(n)$. Let $q(n)$ be a partial recursive function such that $\omega_{q(n)}=\omega_{n}+\{p(n)\}$ for $n \in \operatorname{Dom} \alpha$. Then $\pi_{s}(\alpha, q(n))=\pi(\alpha, p(n))$.

Theorem T 4.3. Let $\alpha$ be semiproductive relative to $p(n)$ and let $\pi_{s}(\alpha, p)$ $\subset \beta \subset \alpha$. Then $\beta$ is semiproductive relative to $p(\dot{n})$ and $\pi_{s}(\beta, p) \subset \pi_{s}(\alpha, p)$.

Proof. Slight modification of the proof of $\mathrm{T} 2.4$.

The first corollary of T 2.4 remains valid if we replace "productive" by "semiproductive" at each of its occurrences and " $\pi(\alpha, p)$ " by " $\pi_{s}(\alpha, p)$. "

Theorem T 4.4. (1) Let $\alpha$ be semiproductive and $\beta$ a recursive set. Then $\beta \subset \alpha$ implies that $\alpha-\beta$ is semiproductive. 
(2) Let $\alpha$ be semiproductive and $\beta$ r.e. Then $\alpha \subset \beta$ implies that $\alpha+\beta^{\prime}$ is semiproductive.

Proof. (1) Let $\gamma$ be a r.e. subset of $\alpha-\beta$, then $\beta+\gamma$ is a r.e. subset of $\alpha$. We can now find a r.e. set $\sigma$ such that $\beta+\gamma C_{+} \sigma \subset \alpha$ (2). Hence $\beta^{\prime}(\beta+\gamma)$ $\subset \beta^{\prime} \sigma \subset \alpha \beta^{\prime}$; this implies $\gamma \subset \beta^{\prime} \sigma \subset \alpha-\beta$ (3), since $\beta^{\prime} \gamma=\gamma$. We conclude from (2) that $\sigma-(\beta+\gamma)=\beta^{\prime} \sigma-\gamma \neq 0$. Thus (3) can be improved to $\gamma C_{+} \beta^{\prime} \sigma \subset \alpha-\beta$. Observe that we can find $\beta^{\prime} \sigma$ because the characteristic function of $\beta^{\prime}$ can be obtained from that of $\beta$. Hence $\alpha-\beta$ is semiproductive.

(2) It follows from $\alpha \subset \beta$ that $\alpha \beta=\alpha$ and $\alpha \beta^{\prime}=0$. Suppose $\delta$ is a r.e. subset of $\alpha+\beta^{\prime}$. Then $\beta \delta \subset \beta\left(\alpha+\beta^{\prime}\right)=\alpha$ and we can find a r.e. set $\sigma$ such that $\beta \delta$ $C_{+} \sigma \subset \alpha$ (4). Let $s \in \sigma-\beta \delta=\sigma \beta^{\prime}+\sigma \delta^{\prime}$ (5). Observe that $s \in \sigma$ implies $s \in \alpha$; hence $s \in \beta$. Thus (5) implies $s \in \sigma \delta^{\prime}$; therefore, $\sigma-\delta \neq 0$. It follows from (4) that $\beta \delta+\delta \subset \sigma+\delta \subset \alpha+\delta$; this implies $\delta \subset \sigma+\delta \subset \alpha+\beta^{\prime}$, since $\delta \subset \alpha+\beta^{\prime}$. Taking into account that $(\sigma+\delta)-\delta=\sigma-\delta$ and that $\sigma-\delta \neq 0$, we conclude $\delta C_{+} \sigma+\delta$ $C \alpha+\beta^{\prime}$. Hence $\alpha+\beta^{\prime}$ is semiproductive.

Definition. Let $\alpha$ be semiproductive. Then Sepr $\alpha={ }_{d f}\left\{n \mid g_{n}(x)\right.$ is a semiproductive function of $\alpha\}$.

Theorem $\mathrm{T}$ 4.5. Let $\alpha$ be semiproductive. Then (1) Dom $\alpha$ is productive, (2) Sepr $\alpha$ is productive.

Proof. (1) The part of the proof of T 2.7 which deals with Dom $\alpha$ requires only the following modification: take $l=p(k)$ instead of $\omega_{l}=\{p(k)\}$.

(2) Suppose $b(t)$ is a recursive function ranging over a nonempty r.e. subset $\beta$ of Sepr $\alpha$. Let $\omega_{n} \subset \alpha$. Then $\omega_{n} C_{+} \omega_{g(b(t), n)} \subset \alpha$ for every $t$. Find a partial recursive function $i(n)$ such that

$$
\omega_{i(n)}=\sum_{t=0}^{\infty} \omega_{o(b(t), n),}, \quad \text { for } n \in \operatorname{Dom} \alpha .
$$

Let $q(n)$ be any semiproductive function of $\alpha$. Then $\omega_{n} C_{+} \omega_{q i(n)} \subset \alpha$ for $n$ $\in$ Dom $\alpha$. Moreover, $q i(n) \neq g_{s}(n)$ for every $s \in \beta$. The conclusion easily follows.

Theorem T 4.6. If $\alpha(m-1)$ red $\beta$ and $\alpha$ is semiproductive, then $\beta$ is semiproductive.

Proof. Let $\alpha$ be semiproductive and $f(n)$ a recursive function such that $f(\alpha) \subset \beta$ and $f\left(\alpha^{\prime}\right) \subset \beta^{\prime}$. Then $f(\alpha) \neq 0$ because $\alpha \neq 0$; hence $\beta \neq 0$. Let $\omega_{n} \neq 0$ and $\omega_{n} \subset \beta$, then we can find a recursive function ranging over $\omega_{n}$; from this function we can find a partial recursive function ranging over $f^{-1}\left(\omega_{n}\right)$. Since $\omega_{n} \subset \beta$ it follows that $f^{-1}\left(\omega_{n}\right) \subset \alpha$. We can now find a r.e. set $\gamma$ such that $f^{-1}\left(\omega_{n}\right) C_{+} \gamma \subset \alpha$; hence $\omega_{n} \cdot \rho(f) \subset f(\gamma) \subset \beta$. Let $t \in \gamma-f^{-1}\left(\omega_{n}\right)$, then $f(t) \in f(\gamma)$ $-\omega_{n} \cdot \rho(f)=f(\gamma)-\omega_{n}$. Thus $f(\gamma) \subset \beta$ and $f(\gamma)-\omega_{n} \neq 0$.

Theorem T 4.7. Among all sets semiproductive relative to $p(n)$ there is a 
smallest one; this smallest set is its own semiproductive center relative to $p(n)$.

Proof. Both proofs of T 2.11 discussed in \$II can be easily modified to prove the present theorem.

The concepts semiproductive function, minimal semiproductive set relative to $p(n)$, and minimal semiproductive set can be defined in an obvious manner. There are denumerably many minimal semiproductive sets and the semiproductive set minimal relative to the semiproductive function $p(n)$ can be defined as the smallest set $\sigma$ (or the intersection of all sets $\sigma$ ) such that for all $n, \omega_{n} \subset \sigma \rightarrow \omega_{p(n)} \subset \sigma$.

Added May 30,1954 . Since this paper was submitted, J. R. Myhill has proved the following two theorems (unpublished):

(1) every productive set is $P$-productive,

(2) every $P$-productive set is completely productive.

The concepts productive, $P$-productive, and completely productive are therefore equivalent. Moreover, every productive set is contraproductive.

Let a contraproductive set be called $P$-contraproductive in case it has a recursive contraproductive function. It can be shown that there exist contraproductive sets which are not $P$-contraproductive.

\section{REFERENCES}

1. J. C. E. Dekker, The constructivity of maximal dual ideals in certain Boolean algebras, Pacific Journal of Mathematics vol. 3 (1953) pp. 73-101.

2. - Two notes on recursively enumerable sets, Proc. Amer. Math. Soc. vol. 4 (1953) pp. $495-501$.

3. S. C. Kleene, Recursive predicates and quantifiers, Trans. Amer. Math. Soc. vol. 53 (1943) pp. 41-73.

4. E. L. Post, Recursively enumerable sets of positive integers and their decision problems, Bull. Amer. Math. Soc. vol. 50 (1944) pp. 284-316.

5. H. G. Rice, Classes of recursively enumerable sets and their decision problems, Trans. Amer. Math. Soc. vol. 74 (1953) pp. 358-366.

NORTHWESTERN UNIVERSITY, Evanston, Ill. 